\section{The Impact of COVID-19 Pandemic in Children With Cancer: A Report From Saudi Arabia}

\author{
Mohammad Alshahrani', Ghaleb Elyamany² (iD), Qanita Sedick² iD, Walid \\ Ibrahim¹, Amal Mohamed', Mohamed Othman'1, Nour Al Thibani', Omar \\ Alsuhaibani², Mohamed Al Amro², Ali Gharawi², Omar Al Sharif', \\ Yasser Elborai ${ }^{1}, 3$ (i), Fahad Alabbas ${ }^{1}$, Amal Binhassan', \\ May AlMoshary ${ }^{4}$, Eman Al Mussaed ${ }^{4}$ and Nawaf Alkhayat ${ }^{1}$
}

${ }^{1}$ Department of Pediatric Hematology/Oncology, Prince Sultan Military Medical City, Riyadh, Saudi Arabia. ${ }^{2}$ Department of Central Military Laboratory and Blood Bank, Prince Sultan Military Medical City, Riyadh, Saudi Arabia. ${ }^{3}$ Pediatric Oncology Department, National Cancer Institute, Cairo University, Cairo, Egypt. ${ }^{4}$ Basic Science Department, College of Medicine, Princess Nourah Bint Abdulrahman University, Riyadh, Saudi Arabia.
Health Services Insights

Volume 13: 1-5

(C) The Author(s) 2020

Article reuse guidelines:

sagepub.com/journals-permissions

DOI: $10.1177 / 1178632920984161$

(S)AGE

\begin{abstract}
In January 2020, the WHO declared the novel coronavirus (2019-nCoV) outbreak as a public health emergency of international concern. Due to the rapid spread of 2019-nCoV, all countries started preventive and precautionary measures to prevent COVID-19 infection spread. These measures limited the population mobility and services provided, which subsequently Impact of on children with cancer and cancer care delivery in the many health centers in Saudi Arabia. We did a cross-sectional study to assess the impact of this outbreak on children with cancer concerning all aspects of life including medical services provided, the specific precautions to prevent spread in cancer patients, mental, psychological effects, and its effect on the quality of life. We collected 204 responses during a survey that assessed the impact on the treatment of cancer children at a tertiary institution during the COVID-19 pandemic. The majority of patients were receiving ongoing chemotherapy for leukemia/lymphoma. The majority of these patients (60.5\%) reported a delay in treatment received due to hospital cancellation of appointments due to the pandemic. Although the majority of patients in our cohort complained of delayed treatment, fortunately, none of the delays led to fatalities. In the context of global lockdowns and physical distancing to help flatten the COVID-19 curve, telemedicine has proved fundamental to keeping patients and their healthcare providers connected and safe. Children also faced multiple other difficulties such as psychosocial issues during the COVID-19 pandemic. Our long-term goals are to develop new programs that will enable children with cancer to emerge successfully during a pandemic.
\end{abstract}

KEYWORDS: COVID 19, cancer, children, mental health, telemedicine

RECEIVED: September 2, 2020. ACCEPTED: December 7, 2020

TYPE: Original Research

FUNDING: The author(s) disclosed receipt of the following financial support for the research, authorship, and/or publication of this article: The SANAD Children's Cancer Support Association with the SANAD Research Grants Program [grant number: RGP-2020-01].
DECLARATION OF CONFLICTING INTERESTS: The author(s) declared no potential conflicts of interest with respect to the research, authorship, and/or publication of this article.

CORRESPONDING AUTHOR: Ghaleb Elyamany, Department of Central Military Laboratory and Blood Bank, Riyadh 11159, Prince Sultan Military Medical City, Riyadh, Saudi Arabia. Email: ghalebelyamany@gmail.com

\section{Introduction}

The coronavirus SARS-CoV-2 causes the disease COVID19, which has become a pandemic and global crisis. It was initially identified in Wuhan, China in December 2019, and has spread worldwide since. ${ }^{1}$ While this infection has proved to be a threat to adults, especially the elderly and people who are already immunocompromised, children seem to be at low risk for serious COVID-19 infections..$^{2-4}$ This includes children who are being treated for cancer, despite being immunocompromised. ${ }^{5}$ However, even though children are minimally affected by the Corona Virus Disease, those patients that do succumb to the virus suffer from severe complications ${ }^{6,7}$ and are also severely impacted by the psychosocial consequences of this pandemic. ${ }^{8-10}$

The approach to combat the virus in most countries all over the world has been more or less similar. Hospitals have resorted to directing most services to patients with COVID-19, reducing or even halting most elective care. Drastic measures such as limiting the number of hospital guests, no entry of individuals into the hospital with symptoms suggestive of COVID-19 infection until a viral test is negative, postponing all follow-up outpatient visits and appointments, avoiding physical contact with patients, isolating children in their hospital rooms and mandatory personal protective gear are some of the measures implemented by hospitals during the pandemic. ${ }^{1}$

Cancer children on chemotherapy require regular outpatient assistance, intermittent hospital admission and support therapy for appropriate therapy delivery. The impact of the COVID-19 pandemic and hospital precautionary measures on this group of patients would therefore have critical consequences because they risk receiving sub-optimal care. The objectives of our study were to assess the impact of the pandemic in children diagnosed with cancer in our Hospital.

\section{Materials and Methods}

We performed a cross-sectional observational study for children diagnosed with cancer at the Prince Sultan Military Medical City in Riyadh. Our survey aimed to assess the impact of the COVID-19 pandemic on children with cancer with regards to the medical service provided, precautionary 

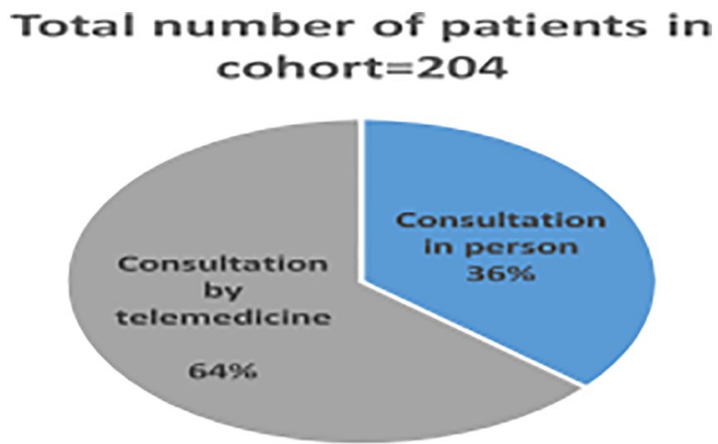

Figure 1. Summary of responses.

measures implemented by the hospital cancer unit to prevent the spread of infection, the acceptance of virtual platforms utilized during the pandemic and the psychological and mental impact of the pandemic in children already diagnosed with a fatal disease. The study was approved by the ethics committee with IRB number: 1374 (HP-01-R079).

To achieve our objective, we designed a voluntary anonymous and targeted questionnaire after extensive literature review of the recent COVID-19 publications concerning cancer patients.

Our inclusion criteria were all children between the ages of 0 to 14 years diagnosed with or recently diagnosed with cancer. Participating parents were asked to complete the booklet type survey questionnaire at the clinic visit during the pandemic or via a virtual platform. Telemedicine conferences with the patients were conducted via video calls to patients at specified times. There were no challenges experienced by doctors with regard to telemedicine conferences with patients. Data were collected on an Excel spreadsheet for analysis. This study was submitted to the Institutional Review Board of Prince Sultan Military Medical City, Riyadh Saudi Arabia, and was approved by the Research Advisory Committee through established procedures. The IRB number allocated is 1374 (HP-01-R079).

\section{Statistical Analysis}

We used descriptive statistics to analyze patient demographic data. We qualitatively analyzed the responses using a pie chart and graphic data generated by the software program.

\section{Results}

\section{Results overview}

We collected 204 responses from cancer patients, 64\% of the data was collected personally in the clinic and 36\% was collected via phone calls and telemedicine (Figure 1). The majority of patients were diagnosed with cancer prior to the pandemic (93\%) and 7\% were diagnosed with cancer during the pandemic.

\section{Cancer type and therapy related results}

Of the 204 cancer patients, $60.4 \%$ of patients were receiving ongoing chemotherapy for leukemia/lymphoma and $39.6 \%$ for

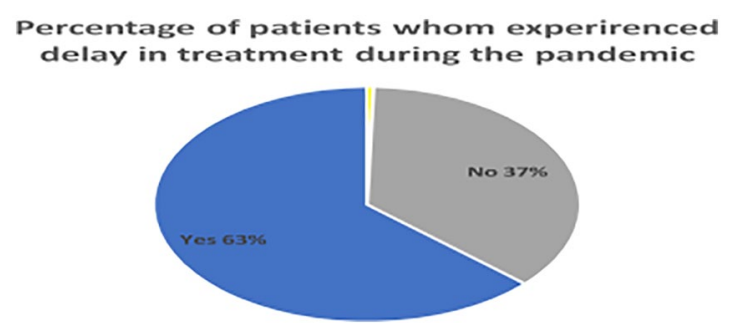

Figure 2. Percentage of delay in treatment of procedure COVID-19 pandemic.

Table 1. Reasons for delay of treatment during the COVID-19 pandemic.

\begin{tabular}{|l|}
\hline Hospital cancellation \\
\hline Hospital with limited capacity \\
\hline Risk of infection increased in hospital environment \\
\hline City lock down and curfew \\
\hline Swab requirements prior to admission \\
\hline Current infection by COVID-19 \\
\hline
\end{tabular}

solid tumors. There were $68.7 \%$ of patients receiving ongoing chemotherapy and the remainder of patients were in remission or on regular follow up.

Of the 204 responses, $63 \%$ of patients reported a delay in treatment received during the COVID-19 pandemic and 37\% of patients reported no delay in treatment (Figure 2).

Patients on chemotherapy experienced treatment delay for various reasons listed in Table 1 . The majority of patients (53.3\%) reported the hospital canceling appointments due to the pandemic precautions as the main reason for treatment or procedure delay during the COVID-19 lockdown and city curfew. There were a total of $30.8 \%$ of patients who reported nonavailability of adequate PPE, lack of cancer support, and shortage of medications as a major challenge faced during the pandemic.

\section{Telemedicine versus hospital visit results}

Of the 204 responses, $65 \%$ reported a preference for telemedicine and virtual clinic visits of which $80 \%$ reported a fear of contracting the virus as the main reason for this preference. The remaining patients preferred telemedicine visits for heterogeneous reasons such as living outside the city or transport difficulties faced during hospital visits.

The minority of patients (35\%) reported a preference for hospital visits due to the perception of more accurate assessments received during personal interaction with the physician (Figure 3).

In addition, $57.3 \%$ of patients strongly agreed to continue attending virtual clinics after the pandemic while 31\% strongly disagreed to continue the virtual clinics. A minority of patients (11.7\%) preferred personal group settings to a mild degree. 
Telemedicine compared to Hospital visits preference

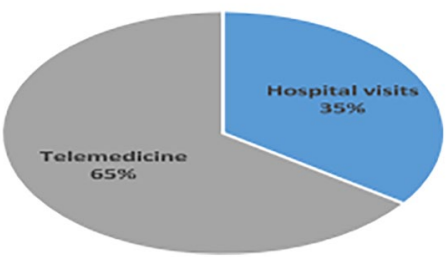

Figure 3. Percentage of telemedicine versus hospital visits.

Patient satisfaction with Hospital COVID 19 precautions

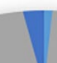

Yes

Figure 4. Patient satisfaction with hospital precautions.

\section{Hospital precautions, safety, and education results}

Fewer patients (40\%) felt safe to visit the hospital during the pandemic compared to $55.3 \%$ of patients who did not feel safe to visit the hospital during the outbreak primarily for fear of contracting the virus in a high risk setting such as hospitals.

All patients adhered to the safety precautions as advised when visiting the hospital and most patients (97\%) were satisfied with the hospital precautions implemented to minimize the risk of infection (Figure 4).

Of the 204 responses, $44.2 \%$ of patients reported having received the patient education materials provided by the hospital while $55.8 \%$ of patients did not receive these materials suggesting an improvement in patient education provided by the hospital as a point of contention.

\section{Childrens mental status results}

The majority of parents (70.2\%) reported that the child had a reasonable understanding of the virus and its potential adverse outcomes. When asked about COVID-19 effects on cancer patients, $90.1 \%$ reported a heightened awareness.

While the majority of patients (87.8\%) of patients did not report an incidence of a family member being affected by COVID-19, $94.8 \%$ of patients were fearful of themselves or a family member contracting the virus. The cancer patients appeared overall more concerned about the complications which may result from contracting the virus than the general public with $53.5 \%$ of positive responses.

\section{Childrens mental health related results}

The majority of patients (81.3\%) of patients experienced an adverse effect on the quality of life during the pandemic predominantly due to limitation in social activities, social isolation, feeling anxious, afraid, and alone.
Table 2. Behavioral and mental characteristics of children with cancer.

\begin{tabular}{|ll|}
\hline BEHAVIOR OF CONCERN & $\begin{array}{l}\text { PERCENTAGE OF } \\
\text { OCCURRENCE IN COHORT }\end{array}$ \\
\hline Anxious about contracting the virus & 96 \\
\hline General anxiety with symptoms & 52.7 \\
\hline Anger & 29.7 \\
\hline Depression & 26.3 \\
\hline Fear & 19.8 \\
\hline Insomnia and nightmares & 12 \\
\hline
\end{tabular}

Table 3. Recommendations to improve hospital service during the pandemic.

Improve pharmacy services during the pandemic to avoid
medication delays
Allow 1 visitor to accompany the patient
Further availability of education material provided by hospital
Delineate clear treatment plan for COVID-19 positive cases
Reduce number of attendees per clinic to reduce waiting time of
patients

There were $67.4 \%$ of parents that were concerned about new onset behavioral occurrences in their children. Behavioral and mental characteristics reported are documented in Table 2.

\section{Survey of patient recommendations}

Patients gave a myriad of recommendations for improving hospital service during the pandemic as listed in Table 3.

\section{Discussion and Recommendations}

We collected 204 responses during a survey that assessed the impact on the treatment of cancer children at a tertiary institution during the COVID-19 pandemic. The majority of patients were receiving ongoing chemotherapy for leukemia/lymphoma. The majority of these patients (60.5\%) reported a delay in treatment received due to the hospital delaying or canceling appointments due to the onset of the coronavirus pandemic. The general consensus emerging from high-risk countries such as Italy and China following the COVID-19 pandemic was to postpone emergency treatments or elective surgery for cancer patients ${ }^{11-14}$; however, the impact of treatment delay on the risk for cancer children is currently lacking.

Although the majority of patients in our cohort complained of delayed treatment, fortunately, none of the delays led to fatalities. Nevertheless, we recommend that a forced delay in treatment due to precautionary measures in the future should be considered on a case by case basis for each patient to avoid critical patients not receiving adequate therapy. 
The survey also highlights the requirement of hospitals to improve the pharmaceutical supply chain to avoid delays in patients receiving critical cancer therapies within critical time. We recommend that pharmacy departments administer homebased therapies in sufficient quantities for patients to minimize the requirement for hospital visits.

Overall, patients did not feel safe visiting the hospital during the pandemic due to fear of contracting the virus in a highrisk setting; however, when visiting the hospital they were satisfied with the hospital precautionary measures implemented. The majority of patients reported a preference for telemedicine, a precautionary measure adopted by most hospitals worldwide. Of the 204 responses, 65.1\% reported a preference for telemedicine and virtual clinic visits of which $80 \%$ reported a fear of contracting the virus as the main reason for this preference. The majority of patients gave positive responses for a continuation of telemedicine post-pandemic.

There is no doubt that the current global COVID-19 crisis has highlighted the significance of leveraging digital approaches to optimize health care in children with chronic illness.

In the context of global lockdowns and physical distancing to help flatten the COVID-19 curve, telemedicine has proved fundamental to keeping patients and their healthcare providers connected and safe. Many practitioners all over the world report that online consultations are proving to be an effective and sustainable patient-care solution. It offers convenient care from the privacy and comfort of one's own home. It has proven invaluable in allowing patients to keep their physical distance while continuing to receive medical care. It is especially useful to ensure continued care for those with chronic conditions, such as children with cancer, to help them overcome any misinformation that may add to their anxiety. It provides healthcare services to people who live in remote areas and also enables worldwide communication among practitioners and patients.

In fact, On March 17, 2020, the US government issued a key temporary waiver for several rules related to Health Insurance Portability and Accountability Act (HIPAA) regulations around telemedicine for both audio and video communications in recognition of the urgent need for telemedicine. ${ }^{15}$ High quality evidence supporting its utility is also well documented. ${ }^{16}$ Digital approaches to pediatric healthcare have also shown therapeutic benefits. A recent meta-analysis concluded that internet-based cognitive behavioral therapy (iCBT) for depression and anxiety in children and adolescents compared to waitlist control groups improved mental health and reduced substance abuse. ${ }^{16}$

We recommend a systematic approach for the physician to adhere to to improve the telemedicine consultation for children with cancer as follows:

- Take a full history of the patient concerning new symptoms or complaints.

- To triage those who need hospital care from those who can be managed via telemedicine.
- Obtain the history of presenting illness, side effects of chemotherapy-associated signs and symptoms.

- Establish medication review.

- Observations: for example, breathing, side effects of chemotherapy.

- Take the vital signs that can be taken by thorough explanation to caregiver.

The most concerning result of the survey was the impact of the pandemic on the mental health status of children. Parents of children reported a heterogeneous array of concerning behavioral changes of children during the pandemic. Children and families experiencing increased anxiety during the pandemic were supported using more frequent telemedicine conferences with the treating physician and referral to psychiatry and psychology were appropriate.

These results are in agreement with other studies in the literature which also found that altered access to health services and economic stress during the COVID-19 pandemic have impacted the mental and physical well-being of youth worldwide, particularly among those with chronic health conditions ( $\mathrm{CHCs}) .{ }^{17}$

Studies indicate that the pandemic could have negative effects on both children's physical and mental health currently and in the long-term. Being quarantined in homes and institutions may result in a greater psychological burden than the physical sufferings caused by the virus. ${ }^{18}$ School closure, lack of outdoor activity, new dietary regimens and sleeping patterns are likely to disrupt children's usual lifestyle and can potentially result in boredom, impatience, agitation, frustration, anxiety, and neuropsychiatric manifestations. ${ }^{19}$ These interlacing challenges of heightened health anxiety, disrupted routines, school closures, family stress and reduced access to physical and psychosocial support experienced in our cohort of children with chronic disease were challenging to overcome in the initial stages of the pandemic.

Children diagnosed with cancer suffer unique problems. While processing the diagnosis of cancer is hard enough, the implications of COVID-19 makes it harder to fully grasp the implications of this new disease on their current ill-health. ${ }^{20}$

Despite these multifactorial challenges faced during the initial stages of the pandemic, there were a multitude of beneficial effects that followed these initial difficulties. The children were able to build resilience to sudden lifestyle changes, increase their knowledge of healthcare technology, and become socially accountable for their actions in addition to having more quality time with families. These effects in children with chronic illness are unanimous with those reported in recent similar studies. ${ }^{17}$

Global research indicates that healthcare for children with cancer should include psychological services to prevent longterm emotional and behavioral problems. ${ }^{21-23}$ This must not be halted due to COVID-19, but rather increased. These services must be delivered in an age-appropriate, developmentally appropriate, and time-sensitive manner. 
To mitigate the psychosocial ill-effects of COVID-19 on children, proactive and targeted interventions can be proposed. Parents, pediatricians, psychologists, social workers, hospital authorities, government, and non-governmental organizations have important roles to play to make the mission successful. It is essential for healthcare professionals to establish multidisciplinary mental health taskforce teams comprised of psychiatrists, psychiatric nurses, clinical psychologists, and other mental healthcare works to provide psychological support to children diagnosed with cancer. Hospitals need to be aware of the social needs of children and make necessary arrangements to maintain communication with their support networks. The use of technology such as audiovisual devices may prove to be useful.

The strengths of our study were that we were able to complete a large amount to questionnaires in a short period using online and physical platforms. The online platforms were conducted via high-speed internet frequency in the majority of cases, which enabled more efficient feedback and communication. There was a large staff of medical doctors and specialists available that connected with patients regularly and therefore was able to extract the most amount of beneficial information. The limitation of our study was that we did not have long term follow up questionnaires of patient's postacute pandemic phase.

\section{Conclusion}

Children with cancer in our institution have faced multiple difficulties including therapy-related and psychosocial issues during the COVID-19 pandemic. However, with proper intervention such as the censored and supervised use of telemedicine and improving the pharmaceutical supply chain to avoid medication delay, children will hopefully emerge from this trying time with unmatched resilience, pliability, solicitude, and cognizance.

\section{Aknowledgements}

The authors would like to thank SANAD Children's Cancer Support Association for their extensive assistance and support of the children afflicted with cancer during COVID-19 pandemic.

\section{Author Contributions}

The Authors MA, GE, QS and NA was responsible for the study design of the manucript. WI, AM, MO and NAT was responsible for data collection. OAS, YE, FA, AB, MAM and EAM was responsible for analysis and interpretation of the data. OA, MAA and AG performed the critical review of the final manucript.All authors contributed to drafting the final manuscript.All authors read and approved the final version of the manucript.

\section{ORCID iDs}

Ghaleb Elyamany (iD) https://orcid.org/0000-0002-6334-4500
Qanita Sedick (iD) https://orcid.org/0000-0003-4499-8266

Yasser Elborai (D) https://orcid.org/0000-0002-5528-0025

\section{Data Accessibility Statement}

The data supporting the findings of this study are available from the corresponding author upon reasonable request.

\section{REFERENCES}

1. World Health Organization. Coronavirus disease (COVID-2019) situation reports-95. WHO; 2020 [Internet]. Accessed May 11, 2020. https://apps.who. int/iris/handle/10665/331905

2. Lu X, Zhang L, Du H, et al. Chinese pediatric novel coronavirus study team. SARS-CoV-2 infection in children. N EnglJ Med. 2020; 382:1663-1665.

3. Zheng F, Liao C, Fan $\mathrm{QH}$, et al. Clinical characteristics of children with coronavirus disease 2019 in Hubei, China. Curr Med Sci. 2020;40:275-280.

4. Sun D, Li H, Lu XX, et al. Clinical features of severe pediatric patients with coronavirus disease 2019 in Wuhan: a single center's observational study. World J Pediatr. 2020;16:251-259. doi:10.1007/s12519-020-00354-4

5. Balduzzi A, Brivio E, Rovelli A, et al. Lessons after the early management of the COVID-19 outbreak in a paediatric transplant and haemato-oncology centre embedded within a COVID-19 dedicated hospital in Lombardia, Italy. Bone Marrow Transplant. 2020;55:1900-1905.

6. Dai M, Liu D, Liu M, et al. Patients with cancer appear more vulnerable to SARS-CoV-2: a multicenter study during the COVID-19 outbreak. Cancer Discov. 2020;10:783-791.

7. Kotecha RS. Challenges posed by COVID-19 to children with cancer. Lancet Oncol. 2020;21:e235.

8. Dubey S, Biswas P, Ghosh R, et al. Psychosocial impact of COVID-19. Diabetes Metab Syndr. 2020;14:779-788.

9. Ghosh R, Dubey MJ, Chatterjee S, Dubey S. Impact of COVID-19 on children: special focus on the psychosocial aspect. Minerva Pediatr. 2020;72:226-235.

10. Vindegaard N, Benros ME. COVID-19 pandemic and mental health consequences: systematic review of the current evidence. Brain Behav Immun. 2020;89:531-542.

11. Lambertini M, Toss A, Passaro A, et al. Cancer care during the spread of coronavirus disease 2019 (COVID-19) in Italy: young oncologists' perspective. ESMO Open. 2020;5:e000759.

12. Liang W, Guan W, Chen R, et al. Cancer patients in SARS-CoV-2 infection: a nationwide analysis in China. Lancet Oncol. 2020;21:335-337.

13. Ferrari A, Zecca M, Rizzari C, et al. Children with cancer in the time of COVID-19: an 8-week report from the six pediatric onco-hematology centres in Lombardi, Italy. Pediatr Blood Cancer. 2020;67:e28410.

14. de Rojas T, Pérez-Martínez A, Cela E, et al. COVID-19 infection in children and adolescents with cancer in Madrid. Pediatr Blood Cancer. 2020;67:e28397.

15. US Department of Health \& Human Services. Notification of enforcement discretion for telehealth remote communications during the COVID-19 nationwide public health emergency. Published 30 March 2020. Accessed 16 November 2020. https://www.hhs.gov/hipaa/for-professionals/special-topics/emergencypreparedness/notification-enforcement-discretion-telehealth/index.html

16. Badawy SM, Radovic A. Digital approaches to remote pediatric health care delivery during the COVID-19 pandemic: existing evidence and a call for further research. JMIR Pediatr Parent. 2020;3:e20049.

17. Serlachius A, Badawy SM, Thabrew H. Psychosocial challenges and opportunities for youth with chronic health conditions during the COVID-19 pandemic. JMIR Pediatr Parent. 2020;3:e23057.

18. Brooks SK, Webster RK, Smith LE, et al. The psychological impact of quaran $\neg$ tine and how to reduce it: rapid review of the evidence. Lancet. 2020;395: 912-920.

19. Lee J. Mental health effects of school closures during COVID-19. Lancet Child Adolesc Health. 2020;4:421.

20. Sprang G, Silman M. Posttraumatic stress disorder in parents and youth after health-related disasters. Disaster Med Public Health Prep. 2013;7:105-110.

21. Kaspers GJL. COVID-19: how will this impact children with cancer, now and in the future? Expert Rev Anticancer Ther. 2020;20:527-529.

22. Parasole R, Stellato P, Conter V, et al. Collateral effects of COVID-19 pandemic in pediatric hematooncology: fatalities caused by diagnostic delay. Pediatr Blood Cancer. 2020;67:e28482.

23. The Lancet Oncology. Safeguarding cancer care in a post-COVID-19 world. Lancet Oncol. 2020;21:603. 